\title{
EDITORIAL
}

\section{Prioritizing Policy Approach and Actions to Address Epidemic of Non Communicable Diseases (NCDs)}

In Bangladesh non communicable diseases (NCDs) historically have not received appropriate attention, although is facing a legacy of huge load of existing and emerging infectious diseases and a cumulative increasing burden of NCDs. NCDs have further burdened the already stretched health system and inflict great cost on the society particularly caused by premature death and disability. According to Bangladesh NCD risk factor survey 2010 there is hardly anyone without a risk factor. About $97 \%$ of population over 25 years of age have at least one risk factor, half the population have two risk factors and about $19 \%$ have 3 or more risk factors. High prevalence and clustering of risk factors in the population warrants urgent mitigation efforts to revert the impending holocaust in very near future.

\section{NCDs as a barrier to development}

The NCD epidemic is exerting an enormous toll in terms of human suffering and inflicts serious damage to human development in both the social and economic realms. The epidemic already extends far beyond the current capacity of country's threshold of resilience. The burden of NCDs is contributing significantly to poverty and has become a major barrier to development and achievement of the MDGs. MDGs that target health and social determinants such as education and poverty are being thwarted by the growing epidemic of NCDs and their risk factors. NCDs are mostly chronic diseases and can lead to continued expenditures that trap poor households in cycles of debt and illness, perpetuating health and economic inequalities, thus forming a vicious cycle whereby poverty exposes people to behavioral risk factors for NCDs. Vulnerable and socially disadvantaged people get sicker and die sooner as a result of NCDs than people of higher socio-economic class. There is strong evidence for the correlation between a host of social determinants, especially education, and prevalent levels of NCDs and risk factors. Treatment for diabetes, cancer, cardiovascular diseases and chronic respiratory diseases can be protracted and therefore extremely expensive. Such costs can force families into catastrophic spending and impoverishment. Household costs for the care of NCDs have a substantial macroeconomic effect. The loss of productivity reduces the society's effective labour force, resulting in reductions in overall economic output. For every $10 \%$ rise in mortality from NCDs, the yearly economic growth is estimated to be reduced by $0.5 \%{ }^{2}$. On the basis of this evidence, the World Economic Forum now ranks NCDs as one of the top global threats to economic development.

\section{Prioritizing interventions for NCDs}

Evidence shows that NCDs are to a great extent preventable. Government has to make difficult choices on how best to allocate resources for health and health care. There is clear evidence that preventive interventions are effective and that improved access to health care can reduce the burden of morbidity, disability and premature mortality ${ }^{3}$. In constructing health policies for the prevention of well-known risks, choices need to be made between different strategies. For instance, preventing small risks in large populations avoid more adverse health outcomes than avoiding large risks in a smaller number of high-risk individuals, leaves ground for discussion. In general it is more effective to give priority to population-based interventions rather than those aimed at high-risk individuals, primary over secondary prevention and controlling distal rather than proximal risks to health. There is a "prevention paradox" which shows that interventions can achieve large overall health gains for the whole population but might offer only small advantages to each individual. This leads to a misperception of the benefits of preventive advice and services by people who are apparently in good health. Evidence shows that population-wide interventions have the greatest potential for prevention particularly in low resource setting like in Bangladesh. There is a huge 
potential for major health gains through sustained multisectoral action involving other ministries and non health agencies concerned with development. Priority should be given to cost-effective interventions for primary rather than secondary prevention for countries like Bangladesh. There are opinions for giving priority to preventing environmental and distal risks to health, such as tackling poor sanitation or inadequate nutritional intakes, rather than the more obvious proximal risks in a causal chain.

Risk factors for NCDs are distributed throughout the society, and they often begin early in life and continue throughout adulthood. Reversing the NCD epidemic requires a comprehensive approach that targets the population as a whole and includes both prevention and treatment interventions. Although feasibility for adopting such interventions depends on factors like the political environment, resource availability, capacity of health-system, community participation and commercial interests of relevant industries.

\section{Priority approaches for NCD prevention}

Bangladesh has developed the National NCD control strategy which has been recently updated ${ }^{4}$. Implementation of major activities from the strategy is desirable. Prerequisite for delivery of immediate priority interventions include, sustained political leadership at the highest levels; support for strengthening the health systems, particularly in the primary health care; monitoring systems and accountability mechanisms for measurement and reporting of progress. UN High-Level Meeting on NCDs created environment for strong highlevel political support for the commitments to tackle the NCD crisis among the political leaders, which is the key to success in the combat against NCDs. Champions and politicians will also need to take the role of steward. Civil society, private sectors and all stakeholders must be brought together. Whole government system works in a compartmentalized way, challenge is to bring them out of silos. Most non-health departments lack the understanding of their role in the prevention of NCDs and perceive this as strictly a health sector's domain, which shows lack of ownership for the issue. In this respect, policy-makers must follow successful approaches to engage non-health sectors based on international experience and lessons learnt. Measuring key areas of the NCD epidemic is crucial to reversing it. Specific measurable indicators must be adopted, like accurate and complete registration of deaths by cause through national registration systems would be the most sustainable mechanism to monitor progress in prevention of NCDs. NCD surveillance must be integrated into national health information systems. Research is needed, firstly, to compare risk perceptions; secondly, to gather data on the frequency of risk factors and their levels in populations; and thirdly, to evaluate the effectiveness and costs of different combinations of interventions.

\section{Health System Response}

Evidence from developed countries shows that launching NCD specific responses within health systems have contributed considerably in declining the NCD trends ${ }^{5}$. Such response is also urgently needed in Bangladesh to curb the steadily rising NCD epidemic. It is also part of the solution to strengthening equity and efficiency of health systems. Ensuring fair health opportunities for everyone is crucial if governments want to uphold the values of equal opportunity, social justice and solidarity. There are growing social inequalities in heart disease, stroke, diabetes, asthma and cancer. The reduction of these health inequities has also an ethical imperative.

\section{Primary Health Care and NCDs}

People with NCDs or at risk of developing NCDs require long-term care and assessments that is proactive, patientcentered, community based and sustainable. The sector wide approach in health care delivery has been adopted in Bangladesh for considerable period which promoted major spendings in primary care. Bangladesh needs to establish and further strengthen an efficient primary care as an integral component of the health systems. World Health Report 2008 provides guidance on the four sets of PHC reforms that are required for providing an effective response to health challenges. These reforms should address universal coverage, service delivery, leadership and governance and public policy.

As there are many competing priority conditions that government needs to address at the primary care level, it is unrealistic to expect government to integrate care for all NCDs into primary care at once. However, there should be hunch for solution for these constrains. As a starting point, a core set of interventions prioritized based on evidence (from home and abroad) can be 
adopted to address the major NCDs, starting at the primary care level, followed by the secondary level and thereon.

\section{Health-systems strengthening}

Strengthening of health-care systems to address NCDs must be undertaken through reorienting existing organizational and financial arrangements and through conventional and innovative means of financing. Capacity should be developed to deliver services for all common diseases during the lifetime, with a patientcentred model of delivery. At first strengthening of primary health care as a part of one point service delivery point that provides the support needed to deliver these critical prevention and treatment services for NCDs is needed. Universal coverage of health care access should be ensured through removal of financial and other barriers to access, particularly for hindered section of the population. Efficient use of resources include subsidy to reduce the costs of accessing services, regulation of user fees in private sectors, health insurance would benefit all health-care users. Curative care based on financial and structural capacity should be considered. Currently health services are yet to be adequate in terms of governance arrangements and health planning processes.

\section{Cost effective interventions}

Preventive strategies focus on the key underlying risk factors for NCDs (tobacco, obesity, physical inactivity and unhealthy diet, and sequeale such as raised blood pressure, blood sugar and cholesterol). Tobacco use alone accounts for one in six of all deaths resulting from NCDs. Implementing four key elements of the WHO Framework Convention on Tobacco Control (tax increases, comprehensive legislation creating smokefree indoor workplaces and public places, health information and warnings about the effects of tobacco, and bans on advertising, promotion and sponsorship) would be a major step. Promoting physical activity and healthy diet through the media and education program and modification of the built environment to promote physical activity can be done. Increase of the price of foods high in saturated fats through taxation, appropriate food labeling and marketing restrictions of unhealthy food products can be achieved through regulatory measures. In addition to tobacco control, reducing indoor air pollution represents the single most important strategy for preventing chronic lung disease, particularly in non-smoking women. Universal access to affordable and good-quality drugs for management of NCDs is an important issue as well.

Finally, incentives and mechanisms to encourage crosssectoral action and coordination are central to sustained progress. Finance ministries need to budget sufficient funds; agriculture ministries to reduce subsidies for harmful crops; trade ministries to enable access to essential medicines; urban planning and transport ministries to create opportunities for greater physical activity; and education ministries to ensure that school environments provide healthy diets through banning the sale and distribution of harmful foods in schools, and promoting health education.

(J Bangladesh Coll Phys Surg 2012; 30: 62-64)

\section{Abul Faiz ${ }^{\mathrm{a}}$, M Ridwanur Rahman ${ }^{\mathrm{b}}$, Md Nazmul} Karimc

a. Professor of Medicine (Retired), Sir Salimullah Medical College, Dhaka, Bangladesh

b. Professor of Medicine, Shaheed Shurwardhy Medical College, Sher-E- Bangla Nagar, Dhaka, Bangladesh

c. National Consultant (NCD), WHO Country Office, Bangladesh

\section{References:}

1. Bangladesh NCD Risk Factor Survey 2010, WHO , Dhaka 2011

2. Stuckler D, Basu S, McKee M. Drivers of inequalities in Millennium Development Goal progress: A statistical analysis. PLoS Med 2010; 7: e1000241.

3. Resolution WHA53.14. Global strategy for the prevention and control of noncommunicable diseases. In: Fifty-third World Health Assembly, Geneva, 22 March 2000. Geneva, World Health Organization, 2000.

4. Strategic Plan for Surveillance and Prevention of NonCommunicable Diseases in Bangladesh, 2011-2015

5. WHO Framework for Action. Everybody`s business: strengthening health systems to improve health outcomes. Geneva, World Health Organization, 2007. 